\title{
Observations of Pre-operative Teamwork and Communication During the Implementation of a City-Wide Surgical Safety Checklist
}

\author{
Terry Leonid Hansen, M.D. ${ }^{1}$, Kyle Goerl, M.D. ${ }^{2}$, Reginald Fears, M.D. ${ }^{3}$, Tim Nguyen, M.D. ,
} Traci Hart, Ph.D. ${ }^{5}$, Paul N. Uhlig, M.D.. M.P.A., F.A.C.S. ${ }^{6}$

${ }^{1}$ University of Arkansas for Medical Sciences

Department of Physical Medicine and Rehabilitation, Little Rock, AR

${ }^{2}$ University of Utah Department of Family and Preventive Medicine, Salt Lake City, UT

${ }^{3}$ University of Kansas Medical Center

Department of Physical Medicine and Rehabilitation, Kansas City, KS

${ }^{4}$ St. Mary's Medical Center, San Francisco, CA

${ }^{5}$ Wichita State University Department of Psychology, Wichita, KS

${ }^{6}$ University of Kansas School of Medicine-Wichita Department of Anesthesiology, Wichita, KS

\begin{abstract}
Background. Use of the World Health Organization's (WHO) perioperative safety checklist has been shown in prior studies to reduce morbidity and mortality. In 2009, the Medical Society of Sedgwick County, Kansas, developed a modified version of the WHO checklist for city-wide implementation. This study evaluated how the checklist was used at a Wichita hospital.

Methods. An observational tool was developed to evaluate time-outs at the beginning of surgical procedures. A convenience sample of cases was evaluated across surgical specialties and procedures. Observations included: 1) when the time-out was done, 2) who led the time-out, 3) which items on the checklist were addressed, 4) how much time was spent, and 5) whether problems were identified or adverse events prevented.

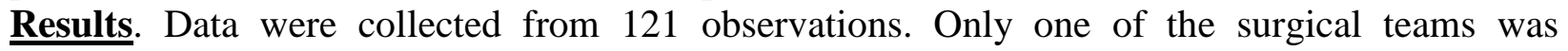
observed to refer directly to the checklist posted in the OR to conduct their time out. The timeout was done before induction (3\%), drape (19\%), incision (77\%), and after incision (1\%). The process was led by the circulating nurse $(92 \%)$, surgeon (7\%), and circulating nurse and surgeon together $(1 \%)$. The percent of completed checklist items was: patient identity $(96 \%)$, procedure (96\%), antibiotics (87\%), site (80\%), allergies (75\%), position (70\%), equipment (60\%), DVT prevention $(50 \%)$, images $(40 \%)$, surgeon concerns $(36 \%)$, and anesthesia provider concerns $(34 \%)$. On average, seven $(\mathrm{SD}=2.5)$ of 11 items on the checklist were addressed. Time spent ranged from less than one minute to five minutes; $78 \%$ took one minute or less. Problems were identified in $7 \%$ of cases. In one case, a wrong site surgery was prevented.

Conclusions. Despite the intention to implement a city-wide surgical safety checklist, the checklist rarely was used in its entirety to conduct the observed time-outs in the subject hospital. Although the checklist was under-utilized, safety benefits were observed from the time-out process. These would likely be enhanced and extended by consistent use of a checklist.
\end{abstract}

KS J Med 2012; 5(4):117-133.

\section{Introduction}

Safety checklists have proven to be a valid and inexpensive cognitive aid to reduce human error and improve teamwork and communication. ${ }^{1}$ High reliability organizations such as the aviation industry, nuclear power industry, and military have long used checklists as a means of safety management. The exceptional safety record of the aviation industry owes much of its credit not to superior pilot skill, but to 
reducing a complex process into a simplified series of checklists to ensure no steps are overlooked. For example, the early form of the pilot's checklist accounted for the four key steps of flying an aircraft: 1) takeoff, 2) flight, 3) before landing, and 4) after the landing. ${ }^{2}$ This simple checklist reduced risks of operator error and minimized the impact of factors related to stress, fatigue, and memory in performing a complex task. ${ }^{3}$

The safety checklist intervention is supported by human factors science and the systems-based approach. ${ }^{4}$ The systems approach emphasizes the importance of team functioning over individual operators. In the systems approach, the operator is not blamed for adverse events; rather the system is analyzed to determine the root cause of errors. After determining the events that led up to an error, defenses are built into the system to prevent recurrence of the previous error as well as future adverse events. ${ }^{5}$ Such an approach has the possibility of elucidating methods that could place the surgical profession on a performance trajectory that could achieve higher goals than may have been possible with the existing model.

In 2008, the World Health Organization (WHO) published guidelines identifying multiple recommended practices to ensure the safety of surgical patients worldwide. ${ }^{4}$ Key among those recommendations was the promotion of a surgical safety checklist. This checklist, evaluated by Haynes et al. ${ }^{6}$, concluded the WHO 19-item surgical safety checklist (see Figure 1; which includes "sign-in", "time-out" and "sign-out" sections) decreased post-surgical complications, deaths, and surgical site infections for non-cardiac patients. The inpatient complication rate was $11 \%$ at baseline and fell to $7 \%$ after implementation $(\mathrm{p}<0.001){ }^{6}$ At baseline, the rate of death was $1.5 \%$ and fell to $0.8 \%$ afterward $(\mathrm{p}=0.003)$.
Approximately 20 million surgeries are performed in the US each year. Applying the mortality reduction observed in the WHO study to this figure, the total deaths related to surgical procedures could fall from about 300,000 to 160,000 . This translates into possibly 140,000 lives saved in the US yearly from the appropriate use of the checklist. This approximation is comparable to estimates presented in other studies.

For many in the healthcare community, the checklist was heralded as a means to ensure a basic minimum safety standard. The surgical safety checklist process implies that every participant of the surgical team has a right and responsibility to communicate if they foresee or notice any errors or problems. Patients who are treated by teams that exhibit less communication are likely to have poorer outcomes. ${ }^{8}$ Properly used, the checklist ensures that critical tasks are carried out and the team is prepared adequately for the operation. ${ }^{9}$

Vats and colleagues ${ }^{8}$ found variability in the way the checklist was implemented noting the informal nature of its execution, unfamiliarity with the checklist among operating room (OR) staff, and uneven support from surgeons and anesthesiologists. Einav et al. ${ }^{10}$ suggested the use of large posters in the OR to guide team members in conducting the checklist. Posters also would serve a secondary purpose of reminding OR staff of commitment to the surgical safety process. Additionally, the checklist has been critiqued for not asking questions early enough to enable corrective action to be taken. ${ }^{10}$ Moreover, some OR teams used the checklist as a tool to ensure completion of a safety process whereas others treated it as a "tick box" exercise. ${ }^{9}$ Further critiques of the surgical safety checklist center on its inapplicability to all surgical specialties. For example, the checklist omitted many 


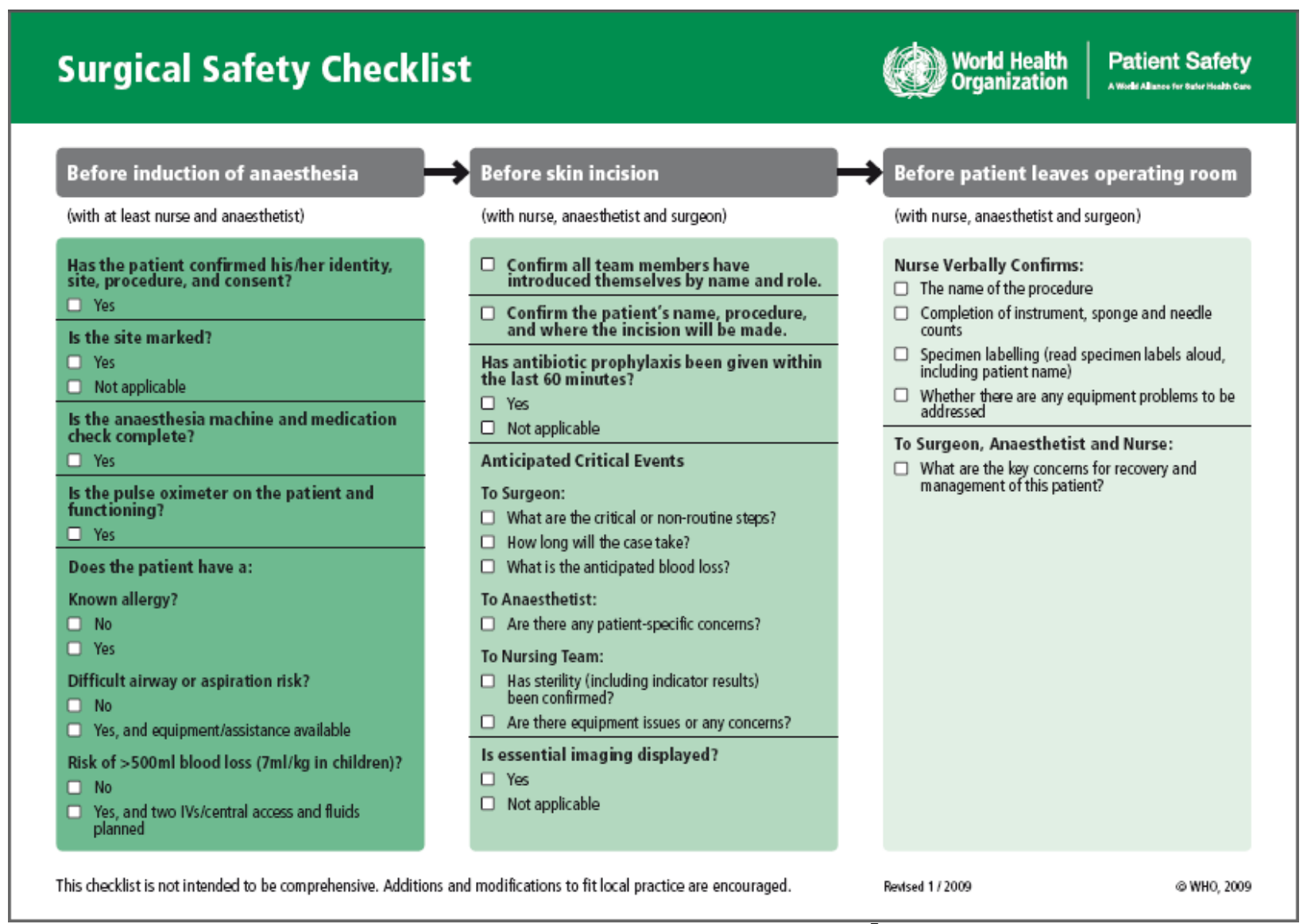

Figure 1. The WHO Surgical Safety Checklist (used with permission). ${ }^{7}$

important safety checks crucial to the safe conduct of cardiac operations such as checks on perfusion equipment and preparation for postoperative intensive care. ${ }^{11}$ Lastly, for all of its safety benefits, the checklist cannot identify and account for competency gaps. ${ }^{12}$

Another factor that influences proper execution of the checklist is teamwork. When teamwork was optimized, the quantity and quality of preoperative briefings and team oriented behaviors increased. ${ }^{8}$ Finally, differences in outlook between clinicians and hospital administrators seemed to hinder attempts to introduce safety-related changes. ${ }^{13}$ The disparity in attitudes regarding safety between OR staff and administration propagates downstream to influence breakdown at the surgical level. ${ }^{14}$ If adherence to the checklist is to be improved, it must be workable and acceptable to all staff. Creating a context where clinicians and managers work together is seen to lead to more success. ${ }^{13}$ The surgical safety checklist, when backed up by good communication and training, results in a flexible yet resilient organization. $^{15}$

The WHO study $^{6}$ evoked a wave of sentiment promoting the surgical safety checklist as a necessary component in the operating room. The Joint Commission (JC), the major accrediting body in the US, followed suit with the WHO and amended its own standards for a perioperative checklist. On July 1, 2004, the JC published, "The Joint Commission Universal Protocol for Preventing Wrong Site, Wrong Procedure and Wrong Person Surgery". ${ }^{16}$ According to the Joint Commission's Universal Protocol requirements for timeout, the team members must confirm the correct patient identity, correct procedure, 
and the correct operation site if applicable. The JC Universal Protocol checklist addressed and augmented many of the areas covered by the first two stages of the WHO checklist (check-in and time-out). The Joint Commission supported the use of the WHO Surgical Safety Checklist, yet the current WHO checklist did not address the standards set forth in the Universal Protocol fully. $^{17}$

Due to impending further regulation from the JC, many surgical centers in the US adopted the use of some form of a surgical safety checklist. The Medical Society of Sedgwick County (MSSC) formed The Wichita Quality Healthcare Collaborative (TWQHC) in 2005 and began discussions on creating its own version of the WHO surgical safety checklist. The MSSC is a 1210 member physician group in Wichita, Kansas, the largest metropolitan area in the state of Kansas. MSSC efforts to create a checklist also were hastened by impending Joint Commission (JC) regulations requiring a surgical safety checklist in the OR.

TWQHC held several meetings at the MSSC which were attended by administrators and OR staff members representing the area's major surgical centers. In March 2009, TWQHC introduced a 9-point consensus checklist to be used within OR time-outs at surgical centers in the greater Wichita area. Their consensus checklist is nearly identical to the WHO's version of the checklist depicted in Figure 1. They concluded that the consensus checklist should be posted in each operating room and referred to by the OR team to conduct their pre-operative briefing.

The surgical teams were advised to refer to the posted checklist each time and respond to the prompts with information read directly from the patient chart. In addition to the $\mathrm{JC}$ 's requirement to verify patient, procedure, and operation site, TWQHC's checklist also complied with the
JC's intent to "ensure that all relevant documents are available and have been reviewed, as well as ensuring blood products, implants, and special equipment are available prior to the start of the procedure and accurately matched to the patient." 18 The observers in this study were tasked with evaluating the manner and consistency with which the pre-procedure safety verification process was performed and inclusive of the required components.

The primary objectives of this study were to: 1) provide observational data regarding OR time-outs while executing the perioperative checklist approved by the Wichita Quality Health Collaborative, 2) discuss the behaviors exhibited by the observed surgical team members and the possible impact on safety outcomes, 3) suggest that the perioperative checklist represents a cultural paradigm shift that may redefine the roles of team members regarding surgical safety.

It was hypothesized that the implementation of the checklist might fail to elicit complete compliance across the observed surgical teams. The full utilization of the surgical safety checklist will require a shift in cultural paradigms; one that replaces the physician-centric culture with a systemsbased, collaborative approach.

\section{Methods}

Intervention. The MSSC began forming The Wichita Quality Healthcare Collaborative in 2005 shortly after The Joint Commission's "Universal Protocol for Preventing Wrong Site, Wrong Procedure and Wrong Person Surgery" became effective on July 1, 2004. During a series of conferences at the MSSC, a committee made up of surgical staff and administrators developed a consensus form of a preoperative checklist. TWQHC officially recommended their checklist for use in operating rooms in the Wichita area in 
March, 2009. It was presented and promoted in the months prior at the subject facility, during OR staff meetings and hospital quality improvement meetings. The data collection process began in April, 2009. All time-outs that were observed in this study occurred weeks after the checklist had been officially implemented at the subject facility.

The TWQHC consensus checklist addresses nine discrete items. One of these items was "special concerns" referring to the whole team. This item was expanded in our observation tool to record role-specific concerns from the surgeon, nurses, and anesthetist. Expanding this item resulted in the 11 measured checklist items referred to in the sections below.

Subjects. One hundred and twenty one observations were conducted at one major medical center in Sedgwick County, Kansas. A random sample of cases was evaluated, between April 2009 and March 2010, across surgical specialties and procedures. This study received scientific review and was approved by the Institutional Review Board.

Procedures. Study personnel arrived prior to the posted surgical start to be in place and capture, by audio recording, the entirety of the event being observed, specifically, the surgical time-out which includes the pre-operative safety checklist (see Appendix). During the observational period, the study personnel collected data on the observation tool.

The observer captured, by audio recording, the entirety of the time-out process and pre-operative safety briefing. During data analysis, the study personnel played back the recorded audio to clarify what was verbalized within the preoperative briefing. Upon completion of the observation tool, the audio recording was erased from the device's memory per approved study protocol. No attempt was made to mark or save any audio recordings. Data elements included a table for OR staff presence and participation, the checklist items, when and how the time-out was conducted, and space for open-ended comments (e.g., concerns being verbalized, "problem solving" responses, and dissenting opinions section on the consensus checklist). Four fourth-year medical students were trained to use the data collection tool. Initially, they trained with video-recorded pre-operative briefings that used a safety checklist (found at www.scoap.org and www.safesurg.org). Then, two study personnel would attend live observations of pre-operative briefings together until their inter-rater reliability exceeded kappa $\geq 0.8$.

\section{Results}

A total of 123 surgical briefings were attended during the course of this study. However, two of the briefings began before induction of anesthesia, therefore, the data could not be applied due to IRB protocol restrictions. Additionally, 25 observations were conducted using the wrong version of the data collection tool and only partial data was retained for analysis.

Observations were conducted at all times throughout the day. However, the most convenient and predictable time was during the first round of operations in a given day. The surgical time-out began when the initiator, typically the circulator nurse, asked the team if they were ready to start the briefing. The circulator typically read the patient's name first, followed by the intended procedure, and continued with some combination of the remaining safety checklist items. Most observations lasted less than five minutes, but observers typically were present in the operating room several minutes before the time-out was initiated.

In one of the 121 eligible evaluations, the checklist posted in the room was referred to specifically during the pre-operative timeout. The other 120 teams did not read from 
nor verbally acknowledge the checklist posted in the OR during the briefing. The items on the checklist were addressed in their entirety in 12 out of $121(10.0 \%)$ timeouts. In two instances (1.7\%), a safety time out was not done at all, violating the Joint Commission's standard.
The average number of items completed on the observation tool checklist was 6.6 of 11 (Std Dev 2.4). The percentage for the number of items completed on the checklists is shown in Figure 2. The completion percentage of each item on the 11 point checklist is shown in Figure 3.

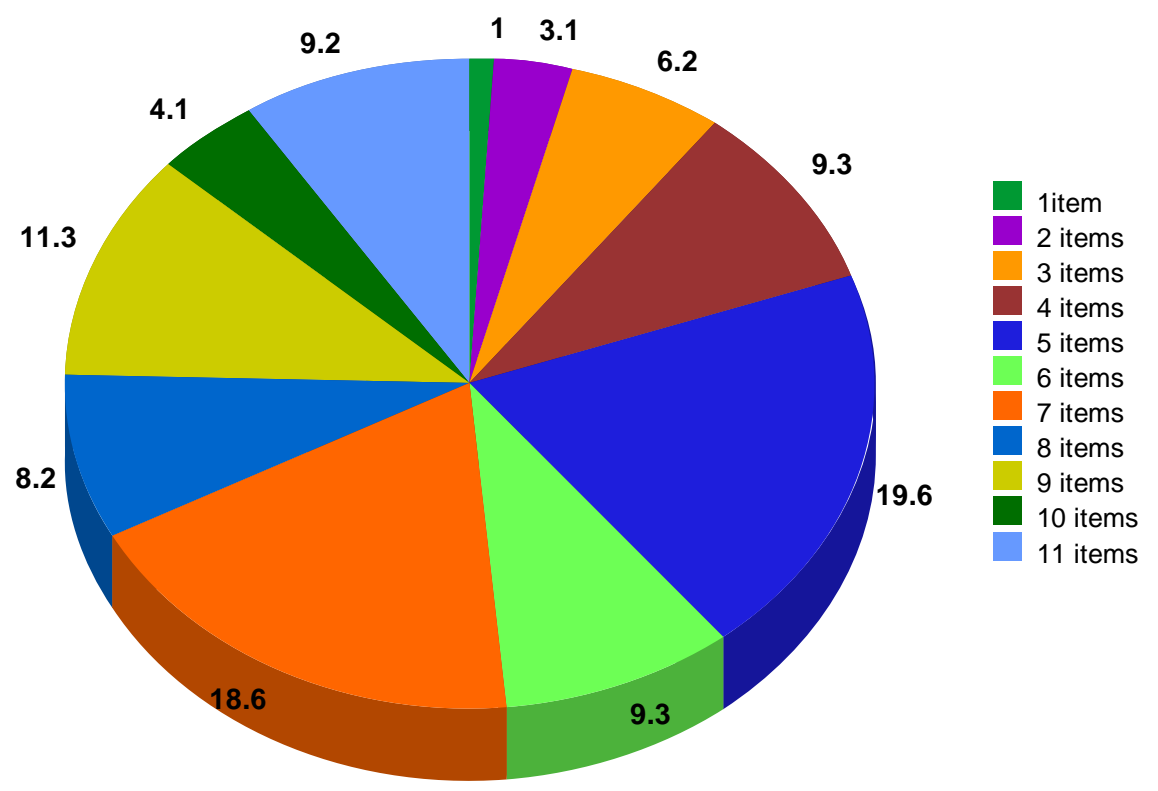

Figure 2. The percentage for the number of items completed on the checklists.

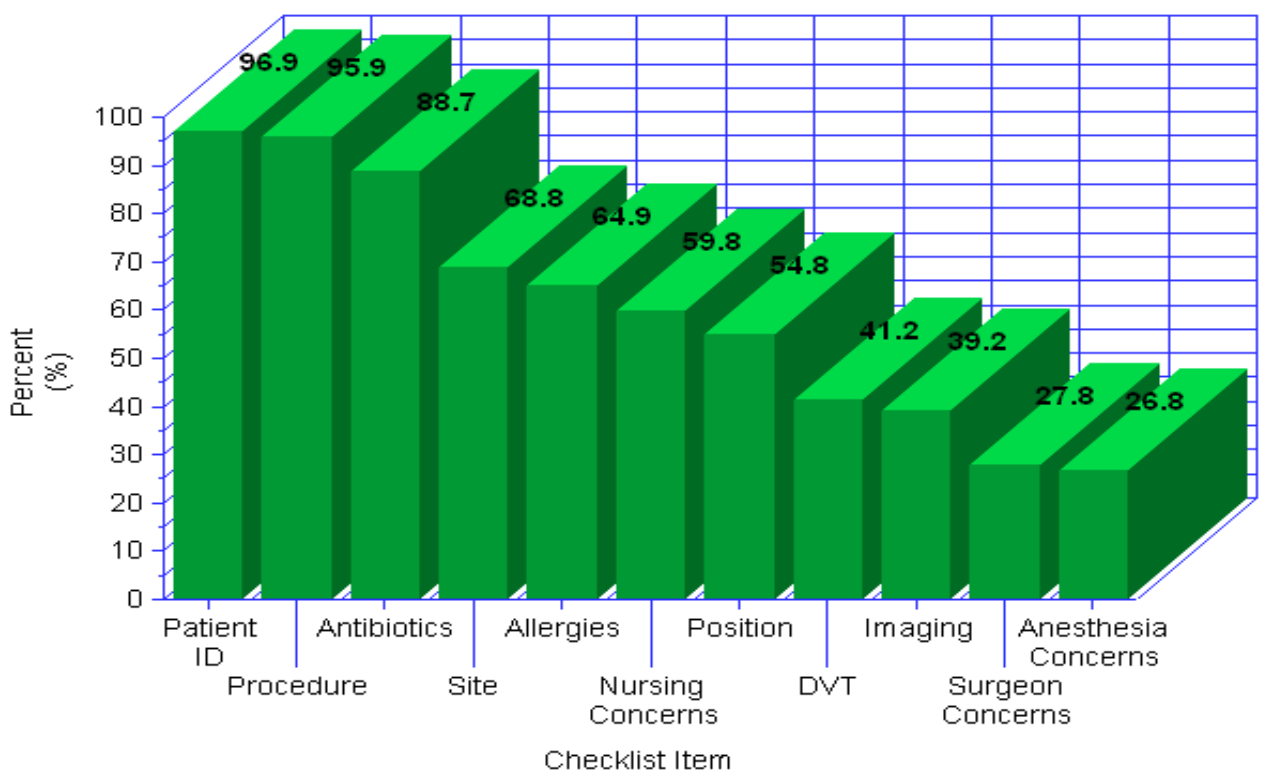

Figure 3. Percentage of checklist items performed. 
Timing of time-out and checklist performance by percentage is shown in Figure 4. The circulating nurse performed the checklist $92.5 \%$ of the time, and the lead surgeon $7.5 \%$ of the time. Eighty-four percent $(83.5 \%)$ of the time the time-out was performed in less than 3 minutes, and in $69.4 \%$ of the cases, it was one minute or less. In 20 observations $(16.5 \%)$, the checklist took longer than five minutes to complete. Attendance and introduction during the time-out were variable: circulating nurse (attended 100\%, introduced $0 \%$ ), attending surgeon (attended $97.5 \%$, introduced $4.2 \%$ ), scrub nurse (attended $97.5 \%$, introduced $0 \%$ ), certified registered nurse anesthetists (attended $90.1 \%$, introduced $0 \%$ ), and the anesthesiologist (attended 35.5\%, introduced $0 \%$ ). The observations of verbal responses by team members are in Table 1 .

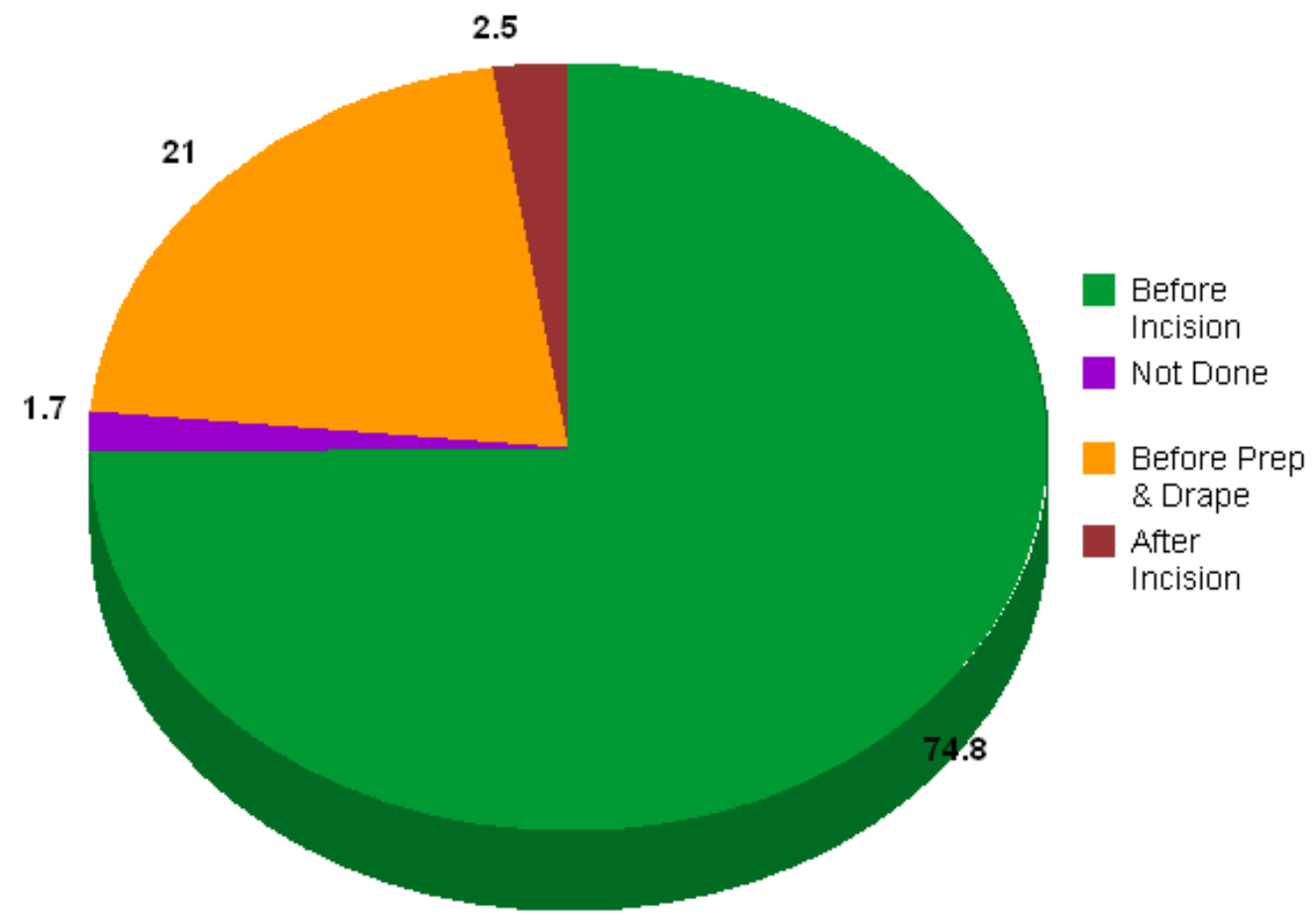

Figure 4. Timing of time-out and checklist performance by percentage.

During the time-out, the leader failed to engage the operating staff members directly in $33.3 \%$ of cases. "Alternatives to the standard procedure," and "problems" were identified in $2.5 \%$ and $6.6 \%$ of cases respectively, but in one case the "problem" identified was that a wrong-sided body part was marked. In one case $(0.8 \%)$, the surgeon verbalized that the operative room was a "safe environment" to voice concerns during the procedure.

\section{Discussion}

The data showed that despite recommendations from the hospital administration, the checklist as a whole was utilized rarely during the pre-operative briefing. The eleven items that we evaluated were addressed fully in only $10 \%$ of the preoperative briefings. In all but one case, the surgical team failed to utilize the posted checklist in the OR and confirm each of the nine items listed there. The tasks completed 
Table 1. Summary of verbal observations.

\begin{tabular}{|c|c|}
\hline Observation Topic & Team Comments (as noted by observers) \\
\hline Problems identified. & $\begin{array}{l}\text { 1. Clarification/uncertainty of a medicine allergy. } \\
\text { 2. Identification of a missing item needed for the procedure. } \\
\text { 3. Confusion by anesthesia over whether or not heparin was given. } \\
\text { 4. Noted the patient as hypothyroid. } \\
\text { 5. Additional antibiotic requested. } \\
\text { 6. Noted by circular nurse that the patient was unsure about their } \\
\text { allergies. } \\
\text { 7. Noted that the surgeon asked for patient to be given more } \\
\text { cefotetan (antibiotic). }\end{array}$ \\
\hline $\begin{array}{l}\text { Changes made to } \\
\text { technology or } \\
\text { approach. }\end{array}$ & $\begin{array}{l}\text { 1. Wrong side of patient initially marked for surgery. } \\
\text { 2. Cell saver requested by surgeon. } \\
\text { 3. Hemavac and nylon suture requested by surgeon. } \\
\text { 4. Decision made about mesh. }\end{array}$ \\
\hline $\begin{array}{l}\text { Action taken on } \\
\text { missing items. }\end{array}$ & $\begin{array}{l}\text { 1. Missing item was located and brought to surgery. } \\
\text { 2. Placed sequential compression devise. } \\
\text { 3. Missing port kit needed for surgery. } \\
\text { 4. Placed Foley catheter. }\end{array}$ \\
\hline $\begin{array}{l}\text { Clarifications requested } \\
\text { on specific items. }\end{array}$ & $\begin{array}{l}\text { 1. Circulator nurse asked the surgeon, "Do you want the large or } \\
\text { small size attachment?" It was verbally resolved by the surgeon. } \\
\text { 2. Anesthesiologist asked the surgeon, "Do you want another dose } \\
\text { of antibiotics?" It was verbally resolved. } \\
\text { 3. Circulator asked the surgeon for clarification of the procedure. } \\
\text { The surgeon responded verbally. } \\
\text { 4. Surgeon asked OR staff, "What could have been done better in } \\
\text { our time out process?" Time out was repeated. } \\
\text { 5. CRNA asked the anesthesiologist, "Shall I replace the } \\
\text { scopolamine path." The answer was, "Yes replace it." } \\
\text { 6. Circulator nurse asked surgeon for procedure clarification. The } \\
\text { surgeon clarified the procedure. } \\
\text { 7. Surgeon asked CRNA, "Were antibiotics given?" CRNA replied } \\
\text { "Yes." } \\
\text { 8. Surgeon asked CRNA, "Do you want Ancef or cefotetan?" and } \\
\text { replied, "Cefotetan." } \\
\text { 9. Surgeon asked circular nurse, "Do we have a Foley in?" } \\
\text { Circulator nurse replied, "Will put Foley in." } \\
\text { 10. Scrub nurse asked circulator nurse, "Can you get disposable } \\
\text { graspers?" Circulator nurse answered, "OK."" } \\
\text { 11. Surgeon asked CRNA, "Were antibiotics given?" CRNA } \\
\text { answered, "Ancef given at } 1449 . " \\
\text { 12. Surgeon asked scrub nurse, "Do we have a port } 2 \text { access site } \\
\text { kit?" Scrub nurse went to retrieve. }\end{array}$ \\
\hline
\end{tabular}


13. Circulator nurse asked CRNA, "Were antibiotics given?" CRNA answered, "Yes, $2 \mathrm{~g}$ of Ancef were given at 0748."

14. Surgeon asked circulator nurse, "Do we have a cell saver?" Nurse obtained cell saver.

15. Circulator nurse asked CRNA, "Did you give Amp and Gent? What time?" CRNA answered, "Amp given at 0718 . Gent given now."

16. Circulator nurse asked CRNA, "Were antibiotics given?" CRNA answered, "Yes, $2 \mathrm{~g}$ of Ancef were given."

17. Surgeon asked anesthesiologist, "Were antibiotics given?" Answered, "Yes, vancomycin was given."

18. Surgeon asked CRNA, "Were antibiotics given?" CRNA answered, "Ancef was given."

19. Surgeon asked circulator nurse, "Did you get her pics (images) up?" Circulator nurse replied, "I will put them up."

20. Circulator nurse asked anesthesiologist, "Were antibiotics given?" Answered, "1 g of cefotetan at 1351."

21. Surgeon asked CRNA, "Do you want antibiotics?" CRNA answered, "She's probably on orals on the floor."

22. Surgeon asked circulator nurse, "Does she have a Foley?" CRNA responded, "No. I will insert Foley."

23. Surgeon asked CRNA, "Did she get antibiotics?" "Yes, 1 g of cefotetan."

24. Surgeon asked circulator nurse, "Does she have SCDS or DVT prophylaxis?" Circulator nurse replied, "Yes, SCDS."

25. CRNA asked surgeon, "Do you want antibiotics?" Surgeon said, "Give Ancef."

26. CRNA asked surgeon, "What antibiotics do you want?" Surgeon answered, "Giving $2 \mathrm{~g}$ Ancef at 0735."

27. Circulator nurse asked surgeon, "What do you want the tourniquet set on?" Surgeon replied, "Set at 300."

28. Scrub nurse asked circulator nurse, "Does he like to use this reciprocator?" Circulator nurse answered, "Yes, that one is what he uses."

29. Surgeon asked CRNA, "Were antibiotics given?" Answered, "Yes."

30. Circulator nurse asked the anesthesiologist, "Were antibiotics given?" Answered, "Yes. Antibiotics were given."

31. Circulator nurse asked surgeon for clarification of procedure terminology. It was clarified by the surgeon. 
with the most consistency were confirmation of patient identity and procedure. One possible explanation is that OR teams were generally quite familiar with these particular tasks due to the JC's mandate (prompting verification the correct patient, procedure, and operating site), effective July 1, 2004, ${ }^{18}$ nearly five years prior to the implementation of TWQHC's checklist. While surgical teams were advised to refer directly to the posted checklist, this behavior was noted in only one case out of 121. The low completion rate of the safety checklist $(10 \%)$ showed that the teams' execution of the checklist did not meet the standards set by their peers and administrators at the medical society meetings.

The JC recommended that, when possible, the patient should be involved in the verification process. ${ }^{19}$ The patient was sedated fully during each of 121 valid timeout observations. Two of the original 123 observations were invalidated by an IRB condition that the patient be unaware of the observer. From a human factors perspective, including the patient in the verification process tends to add to the reliability of the safety briefing. In $73.6 \%$ of cases, the timeout was before incision. This means the patient had been sedated deeply, prepped, and draped. Completing the time-out at that particular juncture bypasses some critical points where errors can occur. For instance, it is possible that the wrong patient could be present or the incorrect side may have been prepped, and the team may not become aware of this until just before incision. Although we were not able to evaluate the briefings in which the patient was included, doing so diminishes the risk of patient misidentification.

Benefits of the safety checklists may include: 1) reducing human error, 2) prompting human interaction, and 3) developing team identity. ${ }^{20}$ Surgical teams navigate many layers of complexity on a daily basis. A systems approach is advocated to deal with this high level of complexity and ultimately curb the effect of human error on patient outcomes. ${ }^{5}$ The safety checklist, if used as intended, clearly falls within the systems approach.

Less clear is what type of human interaction is stimulated by the use of the safety checklist. Our study found that in $33.6 \%$ of the observations the initiator/ leader of the briefing, typically the circulating nurse, did not direct verbal prompts toward any particular staff member. In other words, they did not demand or maintain the full attention of any one particular staff member. A study by Mazzocco et al. ${ }^{8}$ found that lack of communication and team functioning placed patients at a higher risk for death or complications, even after adjusting for the American Society of Anesthesiologists risk category. The timeout checklist, in its intended use, causes the entire team to stop their individual activities and focus together on the primary collective task, verifying a safe and appropriate surgical environment.

The complexity of safety concerns surrounding any one operation are worthy of the undivided attention of all surgical team members. In "The Universal Protocol for Preventing Wrong Site, Wrong Procedure, and Wrong Person Surgery", the Joint Commission stated that during the performance of a time-out "all relevant members of the procedure team [should] actively communicate during the timeout". ${ }^{19}$ Surgical staff may need to employ communication skills, distinct from their technical expertise, to ensure that this behavior occurs reliably. ${ }^{21,22}$

In this study, the anesthesiologist was absent for most of the time-outs. Given that they were ultimately responsible for critical elements that dictate patient vitality (e.g., airway, breathing, and circulation), their presence at the patient safety briefing is 
important. A failure to appreciate expressed concerns regarding patient safety issues discussed during a time-out may delay or impede proper action in emergency situations. The observed surgical department's policy requires that all nurse anesthetists (CRNAs) be supervised by a physician anesthetist. Anesthesiologists are not required to attend time-out briefings explicitly for cases staffed by CRNAs. However, we recommend that they do so, for the very reason that they are required to supervise at all, to ensure that the patient receives the highest quality care possible. In fairness, a single anesthesiologist may be responsible for supervising up to three cases at once, which makes it challenging to avail them at each briefing. Safety measures can be implemented and supported by actions at various levels of administration. Ensuring that the anesthesiologist is present at each briefing may require action on the part of the administrators who determine how each case is scheduled and staffed.

Often in medical practice, time limitations are an issue. This fact may be a source of resistance to the implementation of any safety checklist measures that increase the time commitments of an already overburdened staff. However, the safety checklist has a minimal time commitment; $83.5 \%$ of the briefings took three minutes or less. Within that relatively short time, several critical items can be clarified. Some issues discussed in the safety checklist, if not addressed properly, can cause significant harm to patients. For example, the issue of deep venous thrombosis (DVT) is a considerable risk in the surgical population. In one study, the incidence of DVTs was $3.7 \%$ of all adverse events, and $18 \%$ of those were preventable. $^{23}$ Our study showed that DVT prophylaxis was confirmed in only $41.4 \%$ of briefings. Additionally, site infections are a major contributor to patient morbidity and mortality. The effectiveness of antibiotic administration shortly before skin incision to prevent surgical site infections has been established since the 1960's and has been applied widely in surgical operations. ${ }^{24}$ Our study observed that antibiotic prophylaxis was confirmed in only $86.9 \%$ of pre-operative briefings.

Although the checklist was underutilized, safety benefits were observed from the time-out process. In one observed case, the use of the checklist exposed that the wrong site had been marked prior to the time-out. Had this error persisted, a sentinel event may have occurred in the form of a wrong-site surgery. Other problems identified during time-out discussions, were uncertainty about patient's allergies, missing equipment, and the discovery that additional antibiotics were necessary. Failure to detect and address the aforementioned issues could have resulted in needless harm to patients.

Study limitations. The study was conducted at one Wichita area hospital. Results might have been different had other sites where the MSSC checklist was in use were added. The procedures were selected by convenience and many of the observations were conducted early in the morning when start times were more predictable. Perhaps, some services were under-represented if they were scheduled routinely for afternoon start times.

The observations were completed over a relatively short period. Therefore, a shift in behavior over time could not be measured reliably. Two observers went off protocol and did not use a recording device consistently. However, the briefings were relatively short and predictable and there was little effect on the validity of the observations. Furthermore, the majority of the items registered by the observers were purely objective.

More subjective data from individuals who use the checklist would provide further insight into the behavioral factors that 
dictate its use. To include patient participation, signed informed consent would have been required prior to observing the briefing. We concluded prior to the study that the patient consent process itself would have signaled our observation to the OR staff in a manner that causes them to consciously change their behavior. This change in behavior would have exacerbated the Hawthorne effect (i.e., when participants alter their behavior as a result of being part of an experiment or study), which we reasoned was substantial in this study. As a result, the utilization of the surgical safety checklist may have been, in reality, lower than observed.

\section{Conclusions}

Based on the observed resistance to the safety checklist, a culture change or paradigm shift is necessary to realize the highest possibilities for patient safety. The existing paradigm is one that Atul Gawande refers to as the "Master Builder/Master Physician" model. ${ }^{24}$ Dating back to medieval times, buildings were constructed by a master builder who designed, engineered, and oversaw construction of every aspect of the project from start to finish. Gawande likened this to the existing culture in healthcare which is traditionally physician-centric, generally regarding other

\section{References}

${ }^{1}$ Merry AF. Safer cardiac surgery. J Extra Corpor Technol 2009; 41(4):P43-47. PMID: 20092087.

2 Schamel J. How the Pilot's Checklist Came About. January 31, 2011. Available at: http://www.atchistory.org/History/ checklst.htm. Accessed February 2, 2012.

${ }^{3}$ Hales BM, Pronovost PJ. The checklist - a tool for error management and performance improvement. J Crit Care 2006; 21(3):231-235. PMID: 16990087. providers as ancillary. Full utilization of the safety checklist requires a shift to a systemsbased model rooted in human factors science.

Paradigm shifts are healthy in science because they challenge us to develop and implement models that better deal with reality. Thomas Kuhn, a well-known scientific historian, stated that the new model must seem to resolve some outstanding and generally recognized problem that can be met in no other way. ${ }^{25}$ It also must solve more problems than its predecessor while preserving "a relatively large part of the concrete problem solving activity that has accrued to science through its predecessors." The systems-based model fits Kuhn's description of a revolutionary paradigm. It addresses the global concern about improving patient safety. It better addresses the problems of morbidity and mortality in surgical practice through the use of the safety checklist. ${ }^{6}$ Finally, it preserves our evidence-based clinical methods and confirms that our clinical acumen is applied fully in the care of each patient.

\section{Acknowledgements}

The authors acknowledge Cyndi Sook, R.N. and Holly Fussell for their contributions to this project.

${ }^{4}$ ElBardissi AW, Wiegmann DA, Dearani JA, Daly RC, Sundt TM 3rd. Application of the human factors analysis and classification system methodology to the cardiovascular surgery operating room. Ann Thorac Surg 2007; 83(4):1412-1418. PMID: 17383348.

5 Dankelman J, Grimbergen CA. Systems approach to reduce errors in surgery. Surg Endosc 2005; 19(8):1017-1021. PMID: 16027983. 
${ }^{6}$ Haynes AB, Weiser TG, Berry WR, et al. A surgical safety checklist to reduce morbidity and mortality in a global population. New Engl J Med 2009; 360(5):491-499. PMID: 19144931.

${ }^{7}$ World Health Organization. WHO Surgical Safety Checklist and Implementation Manual. Available at: http://www.who.int/patientsafety/safesurg ery/ss_checklist/en/index.html. Accessed April 7, 2012.

${ }^{8}$ Mazzocco K, Petitti DB, Fong KT, et al. Surgical team behaviors and patient outcomes. Am J Surg 2009; 197(5):678685. PMID: 18789425.

${ }^{9}$ Vats A, Vincent CA, Nagpal K, Davies RW, Darzi A, Moorthy K. Practical challenges of introducing WHO surgical checklist: UK pilot experience. BMJ 2010; 340:b5433. PMID: 20071413.

${ }^{10}$ Einav Y, Gopher D, Kara I, et al. Preoperative briefing in the operating room: Shared cognition, teamwork, and patient safety. Chest 2010; 137(2):443449. PMID: 20133291.

${ }^{11}$ Clark S, Hamilton L. WHO surgical checklist. Needs to be customised by specialty. BMJ 2010; 340:c589. PMID: 20124374.

${ }^{12}$ Mahaffey PJ. Checklist culture. Seductions of the WHO safe surgery checklist. BMJ 2010; 340:c915. PMID: 20179122.

${ }^{13}$ Watts G. Patient safety. Wise before the event. BMJ 2010; 340:c1378. PMID: 20351077.

${ }^{14}$ Taneva S, Grote G, Easty A, Plattner B. Decoding the perioperative process breakdowns: A theoretical model and implications for system design. Int J Med Inform 2010; 79(1):14-30. PMID: 19896893.

${ }^{15}$ Toff NJ. Checklist culture. We need a safety system (and an operations manual). BMJ 2010; 340:c917. PMID: 20179123.

${ }^{16}$ The Joint Commission. Universal protocol. January 13, 2009. Available at: http:// www.jointcommission.org/standards_infor mation/up.aspx. Accessed April 17, 2012.

${ }^{17}$ California Hospital Patient Safety Organization. The Joint Commission Universal Protocol for Preventing Wrong Site, Wrong Procedure and Wrong Person Surgery. Available at: http://www.chpso. org/whosurg/index.asp. Accessed February 2, 2012.

${ }^{18}$ The Joint Commission. Pre-Procedure Verification. January 13, 2009. Available at: http://www.jointcommission.org/stand ards_information/jcfaqdetails.aspx?Standa rdsFAQId=237\&StandardsFAQChapterId $=12$. Accessed February 2, 2012.

${ }^{19}$ The Joint Commission. Speak up: Help avoid mistakes in your surgery. March 19, 2009. Available at: http://www.jointcom mission.org/Speak_Up_Help_Avoid_Mist akes_in_Your_Surgery. Accessed February 2, 2012.

${ }^{20}$ Pronovost PJ, Makary MA, Rowen LC. Evaluation of a preoperative checklist and team briefing among surgeons, nurses, and anesthesiologists to reduce failures in communication--Invited critique. [Discussion.] Arch Surg 2008; 143(1):18.

${ }^{21}$ Yule S, Paterson-Brown S. Surgeons' nontechnical skills. Surg Clinic North Am 2012; 92(1):37-50. PMID: 22269259.

${ }^{22}$ Fletcher G, Flin R, McGeorge P, Glavin R, Maran N, Patey R. Anesthetists' NonTechnical Skills (ANTS): Evaluation of a behavioural marker system. Br J Anaesth 2003; 90(5):580-588. PMID: 12697584.

${ }^{23}$ Gawande AA, Thomas EJ, Zinner MJ, Brennan TA. The incidence and nature of surgical adverse events in Colorado and Utah in 1992. Surgery 1999; 126(1):66-75. PMID: 10418594.

${ }^{24}$ Bratzler DW, Houck PM, Richards C, et al. Use of antimicrobial prophylaxis for major surgery: Baseline results from the National Surgical Infection Prevention Project. Arch Surg 2005; 140(2):174-182. PMID: 157240000. 
${ }^{25}$ Gawande A. The Checklist Manifesto: How to Get Things Right. New York: Henry Holt and Company LLC, 2009. ISBN: 978-0-8050-9174-8.

${ }^{26}$ Kuhn TS. The Structure of Scientific Revolutions. 1st Edition. Chicago: University of Chicago Press, 1962, p. 168.
Keywords: patient safety, preoperative period, surgery, interdisciplinary communication, patient care team 


\section{Appendix}

The Pre-Operative Briefing Observation Tool

Date:

Observer Name:

\begin{tabular}{|c|c|c|c|c|c|c|c|c|c|}
\hline Position & $\begin{array}{l}\text { Circulating } \\
\text { Nurse }\end{array}$ & Surgeon & $\begin{array}{l}\text { Assistant } \\
\text { Surgeon } \\
\text { or } \\
\text { PA/RNFA }\end{array}$ & Anesthesiologist & CRNA & $\begin{array}{l}\text { Scrub } \\
\text { Nurse }\end{array}$ & $\begin{array}{c}\text { Assistant } \\
\text { Scrub } \\
\text { Nurse }\end{array}$ & Patient & $\begin{array}{c}\text { Other } \\
\text { Personnel }\end{array}$ \\
\hline $\begin{array}{l}\mathrm{L}=\text { Lead during } \\
\text { briefing } \\
\text { N/A = Did not lead }\end{array}$ & & & & & & & & & \\
\hline $\begin{array}{l}\text { Person gives a } \\
\text { "problem solving" } \\
\text { response (N/A if not } \\
\text { applicable) }\end{array}$ & & & & & & & & & \\
\hline $\begin{array}{l}\text { Person expressed } \\
\text { dissenting opinion or } \\
\text { frustration w/ checklist } \\
\text { (N/A if not applicable) }\end{array}$ & & & & & & & & & \\
\hline
\end{tabular}




\begin{tabular}{|l||l||l|}
\hline Surgical Checklist for Time-Out: \\
\hline $\begin{array}{l}\text { Confirm the patient's: } \\
\text { Y N Identity Y N Procedure Y N Surgical site Y N Position } \\
\text { Y N Confirms that all essential imaging results for the correct patient are displayed in the } \\
\text { operating room }\end{array}$ \\
$\begin{array}{l}\text { Y Confirms that prophylactic antibiotics have been administered } \leq \mathbf{6 0} \text { min before incision is } \\
\text { made or that antibiotics are not indicated }\end{array}$ \\
$\begin{array}{l}\text { Y N Surgeon reviews critical and unexpected steps, operative duration, and anticipated blood } \\
\text { loss }\end{array}$ \\
Y N Anesthesia staff review concerns specific to patient \\
Y N Nursing staff review confirmation of sterility, equipment availability, and other concerns \\
Y N Patient allergies read from chart \\
Y N Verbally considered DVT prophylaxis
\end{tabular}

\section{During Time-out Briefing: Start Time: $\quad$ End time:}

1. Checklist was done $\square$ Before Induction of Anesthesia $\square$ Before Prep and Drape $\square$ Before Incision $\quad \square$ After Incision $\square$ Not Done

2. To whom did the leader direct the checklist questioning? $\square$ Patient $\square$ OR Staff Member $\square$ Not Centered/Directed

3. Did the team address every point of the checklist? $\square$ NO $\square$ YES (See checklist)

4. Did anyone add some points to the list? $\square$ NO $\square$ YES, please explain:

5. Were any problems identified due directly to a prompt from the checklist? $\square$ NA (if checklist not done) $\square$ NO $\quad \square$ YES, please explain: 
6. Were any last minute changes made to technology or approach from using the checklist? $\square$ NA (if checklist not done) $\square$ NO $\square$ YES, please explain:

7. Was action taken on any missing items? $\quad \square$ NA $\quad \square$ NO $\quad \square$ YES, please explain:

8. Did a discussion of alternatives to the standard procedure arise because of the checklist? $\square$ NA (if checklist not done) $\square$ NO $\square$ YES, please explain:

9. Was a planned technician switch-out verbalized during time-out? $\quad \square$ NO $\quad \square$ YES

10. Who is invoked for clarification of a specific matter?

\begin{tabular}{|l|l|l|l|}
\hline Question & To & From & Resolution \\
\hline & & & \\
\hline & & & \\
\hline & & & \\
\hline
\end{tabular}

11. Is anyone who verbalizes a problem discouraged or reprimanded? $\square$ NA

\begin{tabular}{|l|l|l|l|}
\hline $\begin{array}{l}\square \text { NO } \\
\square \text { YES }\end{array}$ & Proponent:: & Resistor: & Explain: \\
\hline $\begin{array}{l}\square \text { NO } \\
\square \text { YES }\end{array}$ & Proponent:: & Resistor: & Explain: \\
\hline $\begin{array}{l}\square \text { NO } \\
\square \text { YES }\end{array}$ & Proponent:: & Resistor: & Explain: \\
\hline
\end{tabular}

12. Was it ever verbalized that the environment is safe for alternate constructive opinions? $\quad \square$ NO $\quad \square$ YES, please explain: 